\title{
Impact of booster vaccination on the control of COVID-19 Delta wave in the context of waning immunity: application to France in the winter 2021/22
}

Paolo Bosetti ${ }^{1,{ }^{*}}$, Cécile Tran Kiem ${ }^{1,2,{ }^{*}}$, Alessio Andronico ${ }^{1}$, Juliette Paireau ${ }^{1,3}$, Daniel Levy-Bruhl ${ }^{3}$, Lise Alter ${ }^{4}$, Arnaud Fontanet ${ }^{5,6}$ , Simon Cauchemez ${ }^{1}$

1. Mathematical Modelling of Infectious Diseases Unit, Institut Pasteur, Université de Paris, CNRS UMR200o, Paris, France

2. Collège Doctoral, Sorbonne Université, Paris, France

3. Santé publique France, French National Public Health Agency, Saint-Maurice, France

4. Haute Autorité de Santé, Saint-Denis la Plaine, France

5. Emerging Diseases Epidemiology Unit, Institut Pasteur, Université de Paris, Paris, France

6. PACRI Unit, Conservatoire National des Arts et Métiers, Paris, France

* These authors contributed equally to this work.

Correspondence: Simon Cauchemez (simon.cauchemez@pasteur.fr)

Citation style for this article:

Bosetti Paolo, Tran Kiem Cécile, Andronico Alessio, Paireau Juliette, Levy-Bruhl Daniel, Alter Lise, Fontanet Arnaud, Cauchemez Simon. Impact of

booster vaccination on the control of COVID-19 Delta wave in the context of waning immunity: application to France in the winter 2021/22. Euro Surveill. 2022;27(1): pii=2101125. https://doi.org/10.2807/1560-7917.ES.2022.27.1.2101125

Europe has experienced a large COVID-19 wave caused by the Delta variant in winter $2021 / 22$. Using mathematical models applied to Metropolitan France, we find that boosters administered to $\geq 65$, $\geq 50$ or $\geq 18$ year-olds may reduce the hospitalisation peak by $25 \%, 36 \%$ and $43 \%$ respectively, with a delay of 5 months between second and third dose. A $10 \%$ reduction in transmission rates might further reduce it by $41 \%$, indicating that even small increases in protective behaviours may be critical to mitigate the wave.

Most European countries experienced an important rise in severe acute respiratory syndrome coronavirus 2 (SARS-CoV-2) infections and hospitalisations in the autumn of 2021 . In response to this resurgence and to the reported partial decay of immunity, countries have started administering vaccine booster doses, relying on different eligibility criteria. Here, we present modelling analyses assessing different administration strategies for booster doses that informed the recommendations of the French National Immunisation Technical Advisory Group (Haute Autorité de Santé) in the context of Metropolitan France.

\section{Modelling immunity and the impact of vaccines}

We extended a deterministic compartmental model presented in detail by Bosetti et al. [1] (see Supplementary Figure $\mathrm{S}_{1}$ for the model diagram). We account for agespecific mixing patterns [2] and for a lower susceptibility to SARS-CoV-2 infection in children (0-9 and 10-17 years-olds are, respectively, 50\% and 25\% less susceptible than adults) [3,4]. The model considered the epidemic wave caused by the SARS-CoV-2 Delta variant (Phylogenetic Assignment of Named Global Outbreak (Pango) lineage designation (B.1.617.2) and did not capture the future impact of the Omicron variant (B.1.1.529).

Our model explicitly accounted for the decay of vaccine effectiveness [5] (Figure 1). In our baseline scenario, we assumed that after 6 months on average, vaccine effectiveness against infection decreased from $80 \%$ to $50 \%$ [5] and vaccine effectiveness against hospitalisation decreased from $95 \%$ to $85 \%$. In a more pessimistic scenario, vaccine effectiveness against infection decreased to $30 \%$, whereas protection against hospitalisation decreased to $80 \%$ in thoseyounger than 65 years and to $70 \%$ in people 65 years and older. Assumptions regarding vaccine effectiveness are detailed in Supplementary Tables S1 and S2.

We assumed that 7 days after receiving a booster dose, effectiveness against infection and hospitalisation is $95 \%$. After 6 months on average, protection against infection drops to $85 \%$ (protection against hospitalisation remains constant). We also explored a scenario in which the booster confers $99 \%$ protection against hospitalisation. In all scenarios, we assumed that fully vaccinated individuals (with or without a booster dose) and individuals previously infected are half as infectious as individuals with no prior history of infection or vaccination.

We assumed that infected individuals who have not been vaccinated are fully protected against reinfection 


\section{FIGURE 1}

Assumptions regarding SARS-CoV-2 vaccine effectiveness over time
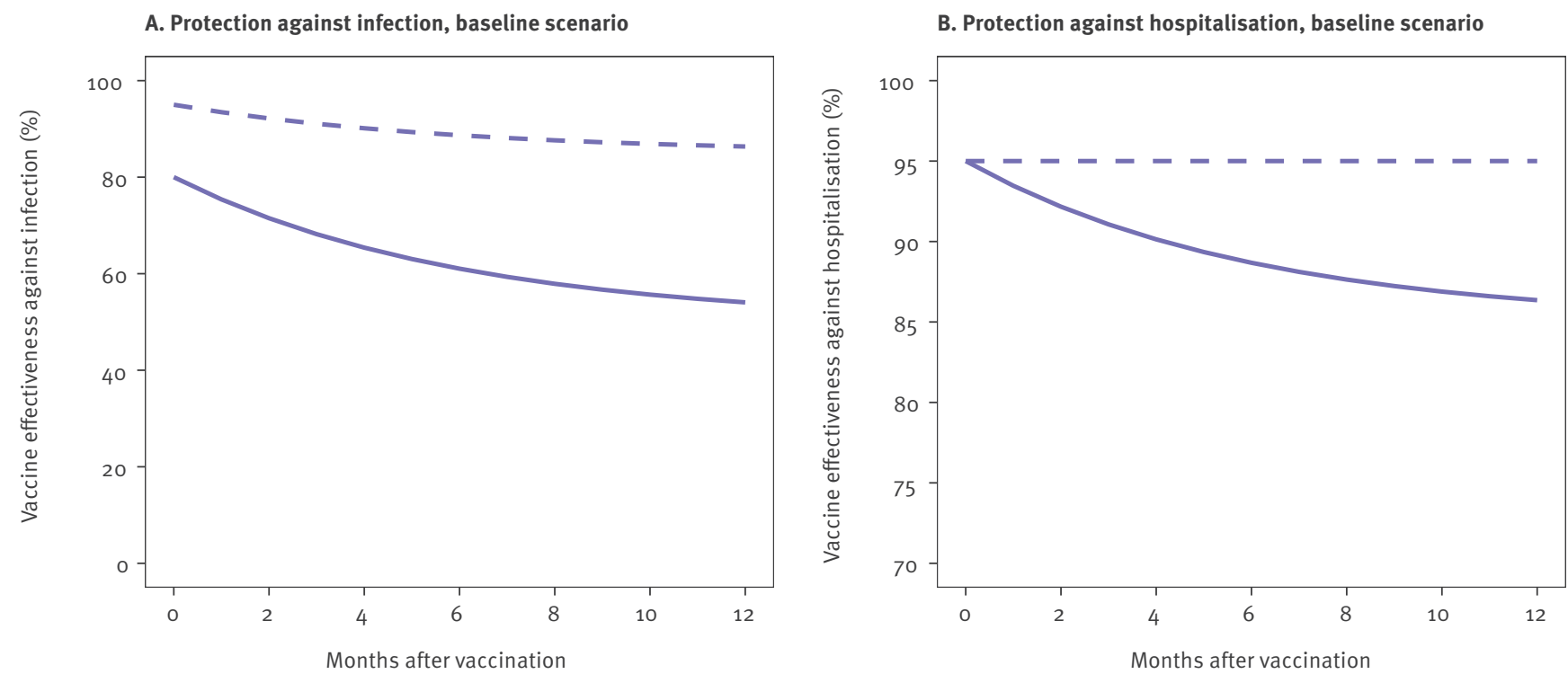

C. Protection against infection, pessimistic scenario

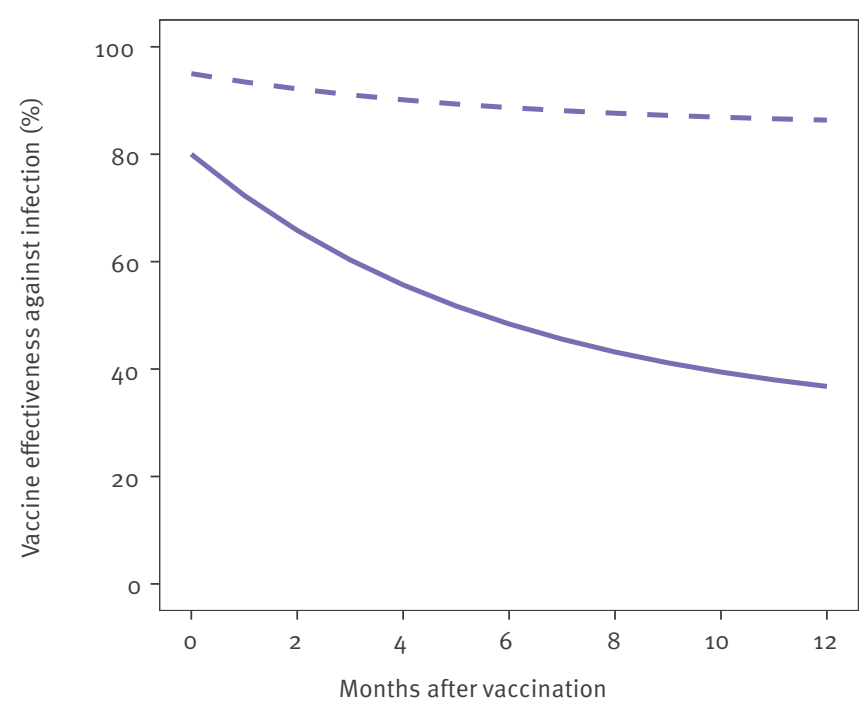

D. Protection against hospitalisation, pessimistic scenario
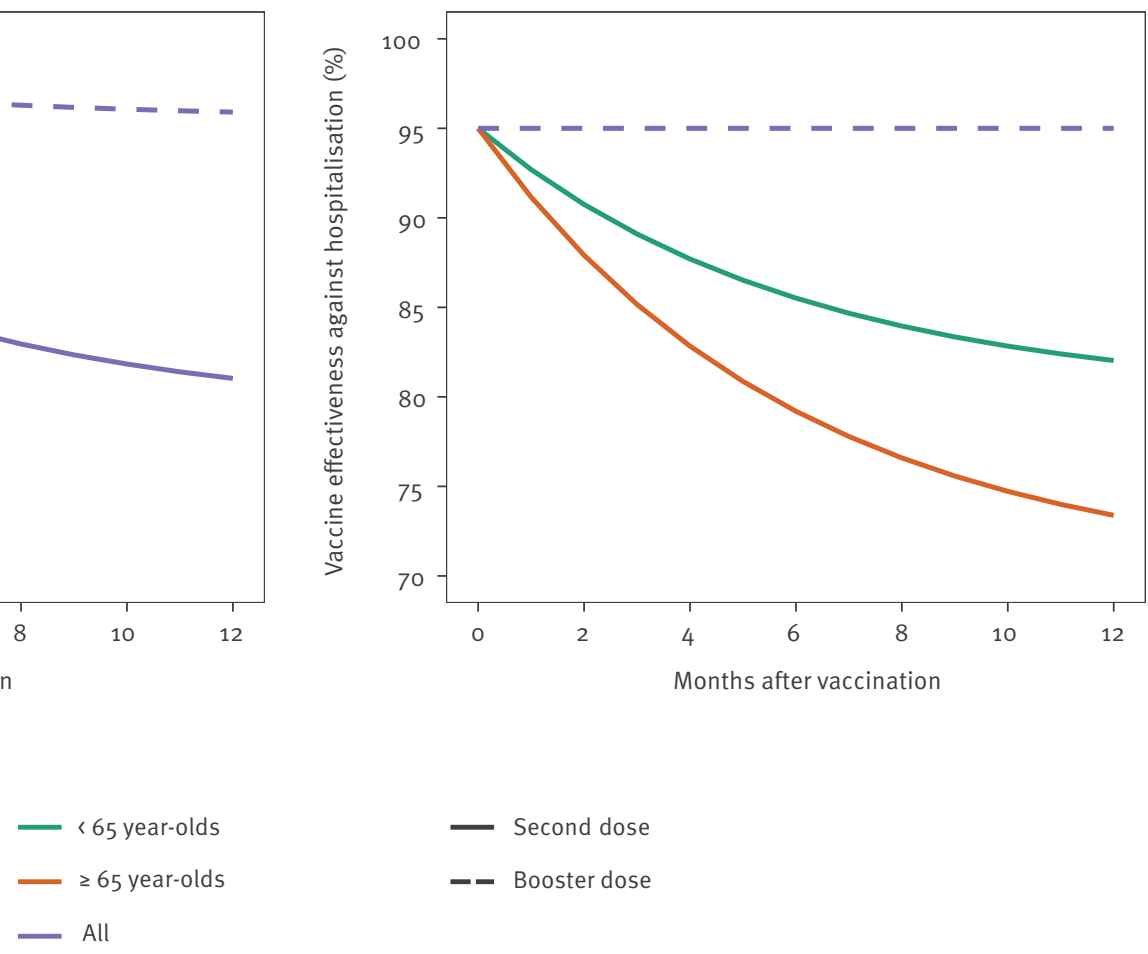

Second dose

- Booster dose

SARS-CoV-2: severe acute respiratory syndrome coronavirus 2.

Note that the y axes are on different scales. 
for 3 months on average. After this, their protection against infection drops to $85 \%$ and, after an additional 6 months on average, to $60 \%$, while protection against hospitalisation drops to $90 \%$ and $85 \%$, respectively.

\section{Administration of vaccine doses}

We assumed that individuals are eligible for a booster dose 5 months after their second dose if they are aged $\geq 65$, $\geq 50$ or $\geq 18$ years. We also explored scenarios where they are eligible 4 or 6 months after their second dose. Among eligible individuals, we assumed that $80 \%$ of $\geq 50$ and $50 \%$ of $18-49$ year-olds accept the booster dose. We also explored a scenario with an acceptance of $95 \%$ for all. We assumed that at most 400,000 or 600,000 doses are administered per day. The future roll-out of second doses was captured with an exponential decrease model (see Supplementary Figure S2 for further detail).

Children aged 5-11 years remained unvaccinated in our baseline scenario. In a sensitivity analysis, this age group was vaccinated from 15 December 2021 at a pace of 50,000 first doses per day with an acceptance of $70 \%$, regardless of the booster roll-out pace.

\section{Epidemiological scenarios during winter}

In our baseline scenario, we assumed that the reproduction number $R_{0}$ (mean number of persons infected by a case accounting for the effect of control measures if there was no population immunity) will remain equal to the one we estimated between 2 and 22 November $2021\left(R_{0}=4.8 ; 95 \%\right.$ credible interval ( $\left.\mathrm{Crl}\right)$ : 4.6-5.0). In sensitivity analyses, we assumed transmission rates decrease by $10 \%$ or $20 \%$ from 1 December 2021 as the population compliance with protective behaviours increases and the government strengthens nonpharmaceutical measures in response to the epidemic progression. All scenarios accounted for seasonal variations ( $33 \%$ amplitude in $\mathrm{R}_{\mathrm{o}}$ between summer and winter) [6]. We assumed that the hospitalisation probability for the SARS-CoV-2 Delta variant is 50\% higher than for Alpha (B.1.1.7) [7], whereas the Alpha variant is $42 \%$ more severe than previously circulating strains [8]. A detailed description of the model and parameters is reported in the Supplement.

\section{Strategies targeting different age groups}

In our baseline scenario, we therefore assumed (i) constant $R_{0}$ from 22 November 2021, (ii) a minimum 5 months delay between the second and third vaccine dose, (iii) a maximum of 400,000 doses administered per day from 1 December 2021 and (iv) a booster acceptance of $80 \%$ and $50 \%$ among $\geq 50$ and $18-49$ year-olds, respectively. We then explored in sensitivity analyses how results changed when we modified these assumptions.

If no booster doses are distributed to the population, we anticipate a peak of 4,140 daily hospital admissions and a cumulative number of 380,000 hospitalisations between 1 November 2021 and 1 May 2022 in
Metropolitan France (Figure $2 \mathrm{~A}$ ). However, if boosters are distributed to those aged $\geq 65, \geq 50$ or $\geq 18$ years, the hospitalisation peak is reduced, respectively, by $25 \%, 36 \%$ and $43 \%$ and the cumulative number of hospitalisations by $23 \%, 33 \%$ and $44 \%$, respectively (Figure 2A).

\section{Strengthening protective behaviours}

When we considered individuals 18 years and older eligible for a booster, reducing $\mathrm{R}_{\mathrm{o}}$ by $10 \%$ and $20 \%$ from 1 December led, respectively, to a reduction in the hospitalisation peak of $41 \%$ and $60 \%$ and a reduction in the cumulative number of hospitalisations of $34 \%$ and $59 \%$, relative to the scenario without reduction in $\mathrm{R}_{\mathrm{o}}$ (Figures 2B-C).

\section{Logistical characteristics of the booster vaccination campaign}

The reduction in the peak number of hospitalisations increased from 35\% for a delay of 6 months between the second and third dose to $43 \%$ for a delay of 4 or 5 months (Figures $3 \mathrm{~A}$ ). Further impact could be achieved by increasing the number of doses administered daily along with acceptance of the booster. For a maximum of 600,000 doses administered daily and an acceptance of $95 \%$ among those 18 years and older, the reduction of the hospitalisation peak and of the cumulative number of hospitalisations is $50 \%$ and $54 \%$, respectively (Figure $3 \mathrm{~B}$ ), compared with $43 \%$ and $44 \%$, respectively, in our baseline scenario.

\section{Vaccine effectiveness}

For more pessimistic assumptions about immunity decay, we expect a higher peak in the absence of boosters, and a larger relative reduction of peak size induced by the booster ( $61 \%$ compared with $43 \%$ in the baseline scenario when $\geq 18$ year-olds are targeted; Figure $3 C)$. A more effective booster ( $99 \%$ reduction against hospitalisation) would also lead to larger reductions ( $55 \%$ when $\geq 18$ year-olds are targeted; Figures 3D).

\section{Vaccination of children}

Vaccinating 5-11 year-old children from mid-December would have limited impact on the hospitalisation peak of the wave in winter $2021 / 22$ ( $2 \%$ reduction compared with a scenario where children are not vaccinated; Figure $3 \mathrm{E}$ ). It would reduce infections and hospitalisations among $0-9$ year-olds by $21 \%$ and $22 \%$, respectively, between 1 November 2021 and 1 May 2022. Assumptions regarding the relative infectivity/ susceptibility in children (Supplementary Table S3) can influence our estimates but the impact on the overall peak in hospitalisations remains low in all scenarios.

\section{Discussion}

Given the reported immunity decay [5], we found that the fast administration of booster doses to adults 18 years and oldervaccinated at least 5 months ago can substantially mitigate the impact of the pandemic wave associated with the SARS-CoV-2 Delta variant in France in winter $2021 / 22$. This result is corroborated by 
Expected impact of different SARS-CoV-2 vaccine boosting strategies on the daily number of hospital admissions, France, July 2021-September 2022

A.

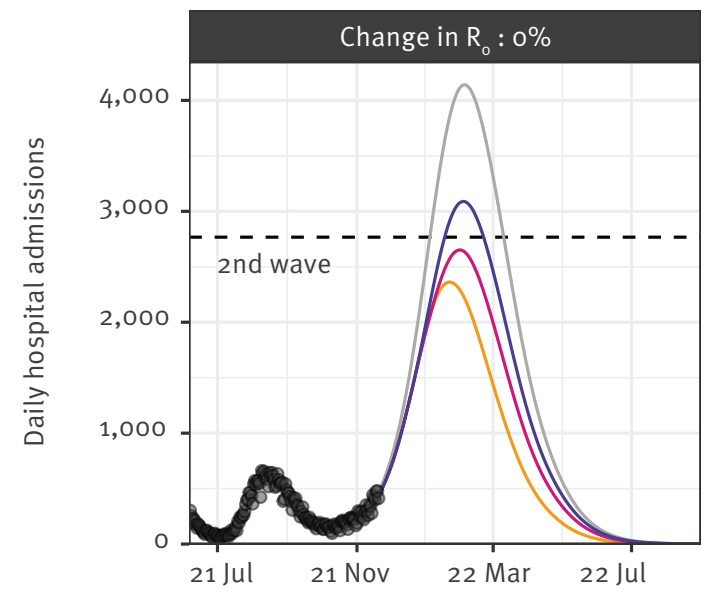

B.

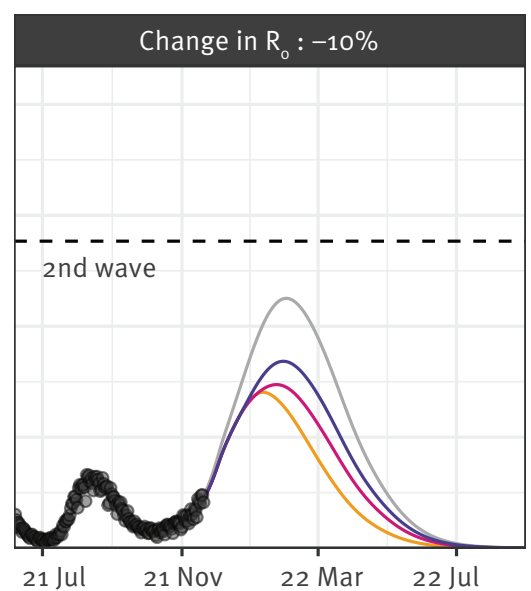

C.

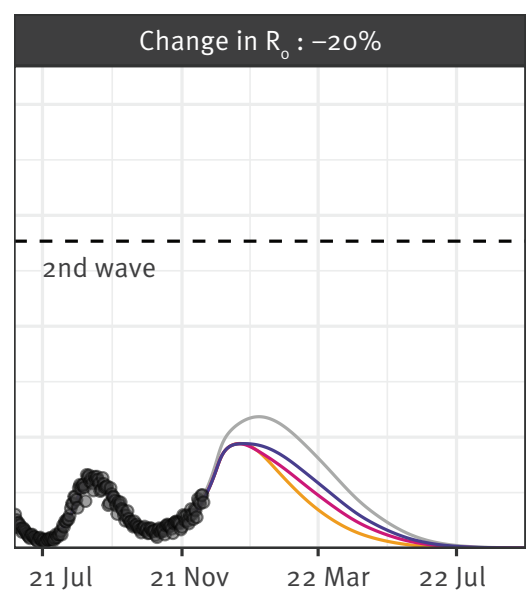

Distribution of boosters

- No one $\quad-\geq 50$ years

$-\geq 65$ years $-\geq 18$ years

SARS-CoV-2: severe acute respiratory syndrome coronavirus 2.

Daily number of hospital admissions assuming transmission rates from 1 December 2021 remain unchanged (panel A), are reduced by $10 \%$ (panel B) or are reduced by $20 \%$ (panel C). We explored strategies where booster doses are not distributed, distributed in those aged 65 and older only, those aged 50 and older and those aged 18 and older.

the experience in Israel, where a large pandemic wave could be controlled with such an approach [9].

Administering boosters to all adults has a larger impact than targeting older adults only because of (i) the important decay of protection against infection and (ii) the important contribution of young adults to SARSCoV-2 spread [10]. In this context, increasing their protection reduces community transmission, indirectly protecting frail individuals. Small reductions in $R_{0}$ due to the strengthening of protective behaviours can have an important effect on epidemic dynamics.

While our results may inform recommendations in other European countries, they are sensitive to country-specific features. Firstly, France has achieved high twodose vaccine coverage (ca $80 \%$ of teenagers and $90 \%$ of adults). In countries with lower vaccine coverage, boosting vaccinated individuals should have a more limited impact, since unvaccinated individuals contribute more to disease spread and hospitalisations. Secondly, the French population was mostly vaccinated with the SARS-CoV-2 Comirnaty vaccine (BNT162b2 mRNA, BioNTech-Pfizer, Mainz, Germany/New York, United States). For vaccines characterised by larger immunity decay, boosting may lead to larger gains. Finally, the impact of logistical features (e.g. delay between the second and the third dose, maximum number of doses distributed daily) will depend on the timing of second dose distribution relative to the current wave. For example, under the assumption that the vaccine boost has the same impact on the immune system when administered after 4, 5 and 6 months, we found that reducing the delay between doses can provide substantial gains in France because many French people were vaccinated in Summer 2021 (see Supplementary Figure S3 depicting the metropolitan French population eligible through time). Those gains might be more limited if countries achieved high vaccine coverage at a different time.

We find that vaccinating children from mid-December would have little impact on the current hospitalisation wave. This result reflects the late timing of this vaccination with respect to the wave. The impact of vaccinating children could have been substantial if it had started earlier (Supplementary Figure S4). It is therefore important to anticipate the impact beyond the current Delta wave, particularly with the rise of the Omicron variant [11].

We investigated the impact of boosting on the Deltadriven pandemic wave in winter 2021/22. The emergence of the Omicron variant is a cause for concern 


\section{FIGURE 3}

Sensitivity analyses exploring different SARS-CoV-2 booster and vaccine eligibility criteria, booster and vaccine effectiveness and booster acceptances, France, July 2021-September 2022

A. Different delays until booster eligibility
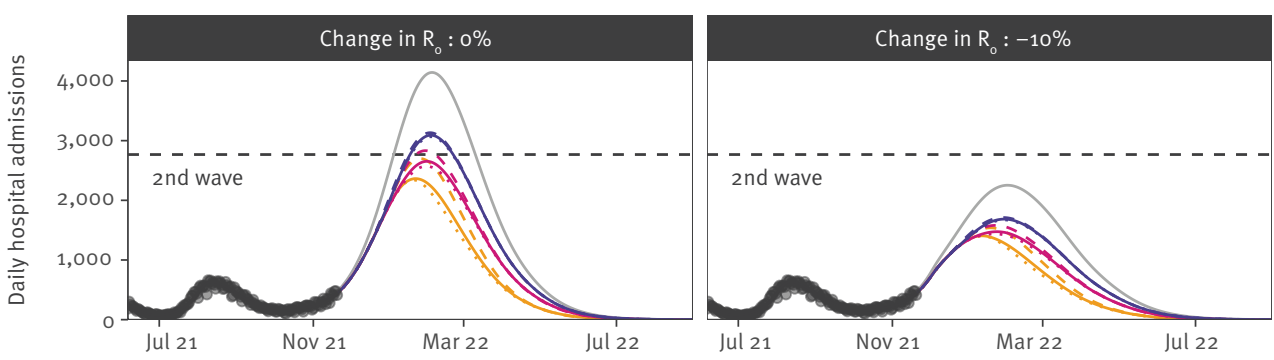

B. Different assumptions about booster acceptance
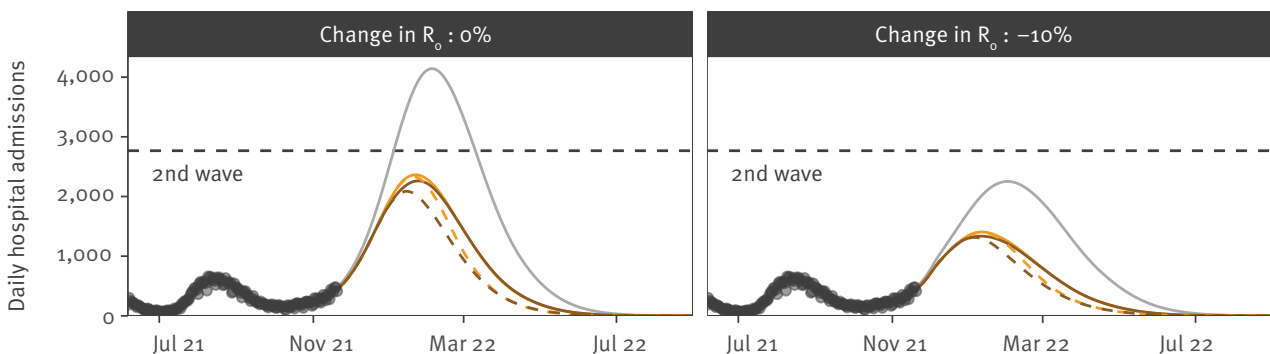

C. Different assumptions about waning of protection

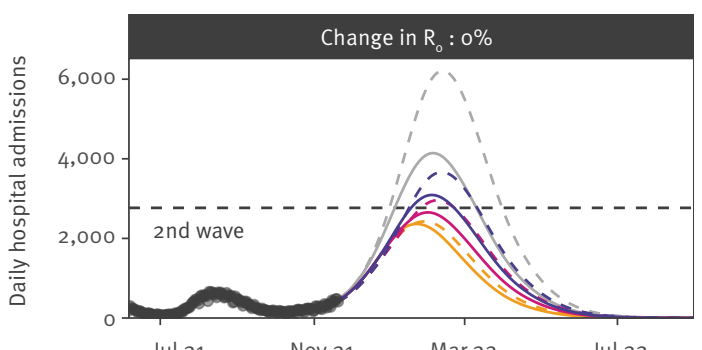

Jul 21

Nov 21
Jul 22

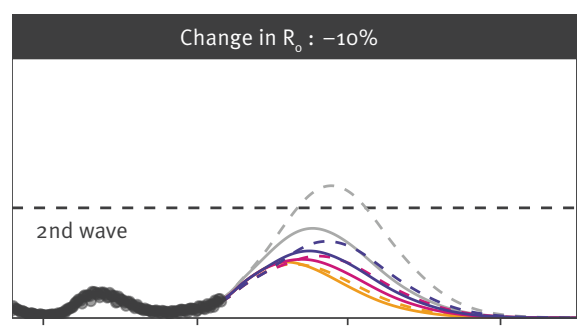

Jul 21
Distribution of boosters

- No one

- $\geq 65$ years

— $\geq 50$ years

$\longrightarrow \geq 18$ years

Delay 2nd dose - eligibility

- - 6 months

- 5 months

… 4 months

Booster roll - out pace $(\times 1,000)$

- 0

$-400$

$-600$

\section{Acceptance booster}

$\begin{array}{ll} & 80 \% \geq 50 \text { years } \\ - & 50 \% 18-49\end{array}$

- $95 \% \geq 18$ years

Distribution of boosters

- No one

— $\geq 65$ years

— $\geq 50$ years

_ $\geq 18$ years

Assumptions waning protection

- Reference

- - Pessimistic

Distribution of boosters

- No one

— $\geq 65$ years

$-\geq 50$ years

— $\geq 18$ years

Assumptions effectiveness boosters

- Reference

- - Higher

Distribution of boosters

- No one

- $\geq 65$ years

$-\geq 50$ years

$-\geq 18$ years

Vaccination of 5-11 year-olds

- No

- Yes

SARS-CoV-2: severe acute respiratory syndrome coronavirus 2.

Panel A: Impact of changing the delay between receiving a second dose and being eligible for a booster. Panel B: Impact of an increased acceptance of booster doses and/or a faster roll-out of boosters. Panel C: Impact of more pessimistic assumptions about the waning of protection conferred by vaccination. Panel D: Impact of a higher effectiveness of booster doses. Panel E: Impact of the vaccination of children. In panel B, the trajectories are presented under the assumption that booster doses are distributed in those 18 years and older. 
[11] and will add to the burden anticipated for the Delta variant. The impact of Omicron will depend on its characteristics (transmissibility, severity, immune escape). In any case, measures available to mitigate the Delta wave (booster doses and strengthening of protective behaviours) will also help delay and mitigate this impact.

\section{Conclusion}

The rapid roll-out of booster doses to the population 18 years and older can reduce the impact of the wave caused by the SARS-CoV-2 Delta variant considerably. Small reductions in transmission rates (e.g. from the adoption of protective behaviours) can substantially reduce the stress on the healthcare system.

\section{Acknowledgements}

Funding: We acknowledge financial support from the Investissement d'Avenir program, the Laboratoire d'Excellence Integrative Biology of Emerging Infectious Diseases program (grant ANR-10-LABX-62-IBEID), HAS, Santé Publique France, the EMERGEN project (ANRS0151), the INCEPTION project (PIA/ANR-16-CONV-0005), the European Union's Horizon 2020 research and innovation program under grant 101003589 (RECOVER) and 874735 (VEO), AXA and Groupama.

\section{Conflict of interest}

None declared.

\section{Authors' contributions}

PB, CTK and SC conceived the study. PB, CTK, AA and JP performed the analyses. DLB, LA and AF helped define model assumptions and interpret the results. PB, CTK and SC wrote the first draft. All authors contributed to revisions of the manuscript.

\section{References}

1. Bosetti P, Tran Kiem C, Andronico A, Colizza V, Yazdanpanah $Y$, Fontanet $A$, et al. Epidemiology and control of SARS-CoV-2 epidemics in partially vaccinated populations: a modeling study applied to France. Paris: Institut Pasteur; 2021. Available from: https://hal-pasteur.archives-ouvertes.fr/ pasteur-03272638v2

2. Béraud G, Kazmercziak S, Beutels P, Levy-Bruhl D, Lenne $\mathrm{X}$, Mielcarek $\mathrm{N}$, et al. The French connection: the first large population-based contact survey in France relevant for the spread of infectious diseases. PLoS One. 2015;10(7):e0133203. https://doi.org/10.1371/journal.pone.0133203 PMID: 26176549

3. Viner RM, Mytton OT, Bonell C, Melendez-Torres GJ, Ward J, Hudson L, et al. Susceptibility to SARS-CoV-2 infection among children and adolescents compared with adults: a systematic review and meta-analysis. JAMA Pediatr. 2021;175(2):143-56. https://doi.org/10.1001/jamapediatrics.2020.4573 PMID: 32975552

4. Davies NG, Klepac P, Liu Y, Prem K, Jit M, Eggo RM, CMMID COVID-19 working group. Age-dependent effects in the transmission and control of COVID-19 epidemics. Nat Med. 2020;26(8):1205-11. https://doi.org/10.1038/s41591-0200962-9 PMID: 32546824

5. Tartof SY, Slezak JM, Fischer H, Hong V, Ackerson BK, Ranasinghe ON, et al. Effectiveness of mRNA BNT162 b2 COVID-19 vaccine up to 6 months in a large integrated health system in the USA: a retrospective cohort study. Lancet. 2021;398(10309):1407-16. https://doi.org/10.1016/S0140 6736(21)02183-8 PMID: 34619098
6. Collin A, Hejblum BP, Vignals C, Lehot L, Thiébaut R, Moireau $R$, et al. Using population based Kalman estimator to model COVID-19 epidemic in France: estimating the effects of nonpharmaceutical interventions on the dynamics of epidemic. medRxiv. 2021.07.09.21260259. preprint. https://doi.org/10.1 101/2021.07.09.21260259 https://doi.org/10.1101/2021.07.09 21260259

7. Twohig KA, Nyberg T, Zaidi A, Thelwall S, Sinnathamby MA, Aliabadi S, et al. , COVID-19 Genomics UK (COG UK) consortium. Hospital admission and emergency care attendance risk for SARS-CoV-2 delta (B.1.617.2) compared with alpha (B.1.1.7) variants of concern: a cohort study. Lancet Infect Dis. 2022;22(1):35-42. https://doi.org/10.1016/S14733099(21)00475-8 PMID: 34461056

8. Bager P, Wohlfahrt J, Fonager J, Rasmussen M, Albertsen M, Michaelsen TY, et al. , Danish Covid-19 Genome Consortium. Risk of hospitalisation associated with infection with SARSCoV-2 lineage B.1.1.7 in Denmark: an observational cohort study. Lancet Infect Dis. 2021;21(11):1507-17. https://doi. org/10.1016/S1473-3099(21)00290-5 PMID: 34171231

9. Bar-On YM, Goldberg Y, Mandel M, Bodenheimer O, Freedman L, Kalkstein N, et al. Protection of BNT162 b2 Vaccine Booster against Covid-19 in Israel. N Engl J Med. 2021;385(15):1393400. https://doi.org/10.1056/NEJMoa2114255 PMID: 34525275

10. Tran Kiem C, Bosetti P, Paireau J, Crépey P, Salje H, Lefrancq $\mathrm{N}$, et al. SARS-CoV-2 transmission across age groups in France and implications for control. Nat Commun. 2021;12(1):6895. https://doi.org/10.1038/s41467-021-27163-1 PMID: 34824245

11. Pulliam IRC, van Schalkwyk C, Govender N, von Gottberg A, Cohen C, Groome MJ, et al. Increased risk of SARS-CoV-2 reinfection associated with emergence of the Omicron variant in South Africa. bioRxiv. 2021.11.11.21266068. preprint. https://doi.org/10.1101/2021.11.11.21266068 https://doi. $\operatorname{org} / 10.1101 / 2021.11 .11 .21266068$

\section{License, supplementary material and copyright}

This is an open-access article distributed under the terms of the Creative Commons Attribution (CC BY 4.0) Licence. You may share and adapt the material, but must give appropriate credit to the source, provide a link to the licence and indicate if changes were made.

Any supplementary material referenced in the article can be found in the online version.

This article is copyright of the authors or their affiliated institutions, 2022. 